\title{
Simulation research of the buffering capacity of non-convex particle system under impact loading
}

\author{
Qi Zhang ${ }^{1, *}$, Siqiang Wang ${ }^{1}$, and Meiying $\mathrm{Hou}^{2}$ \\ ${ }^{1}$ Department of Mechanics, Taiyuan University of Technology, Taiyuan, China \\ ${ }^{2}$ Key Laboratory of Soft Matter Physics, Institute of Physics, Chinese Academy of Science,Beijing, China
}

\begin{abstract}
The behavior of granular materials under the action of external force is a fundamental problem for the dynamics of granular media. This paper investigates the buffering capacity of three-dimensional non-convex granular beds subjected to vertical impact using the discrete element methods. The results display the particle shape significantly affects the buffering capacity of the equal mass granular bed. And the influence of aspect ratio of non-convex particles on buffering capacity exhibits an obvious non-monotonicity. The functional mechanism of inter-particle friction is discussed. It is showed that the shape of the non-convex particles has a great effect on the contact number of granular system and probability density functions of inter-particle forces.
\end{abstract}

\section{Introduction}

The behavior of granular systems is rather complex due to their dissipation and many-body nature[1-2]. At present, the most research of granular materials focus on the spherical particles system, while particles in nature are generally non-spherical[3]. Compared to spherical particles, the non-spherical particles are significant differences in the rheological behavior and structural properties like viscosity, yield stress and packing density, especially for non-convex particles system which could exhibit an effective cohesion through the interpenetration between particles[4-6].

In recent years, the impact problems of granular matter become particularly important in the field of materials engineering, soil mechanics and fracture mechanics[7-10]. Granular materials demonstrate many unique properties under external impact loading by means of energy dissipation. Particles could extend the time of impact by breaking and recombination of internal force chain network, thereby reducing the impact strength. Particle damper composed of spherical particles is widely used in engineering and industrial application. While dolosse made from concrete with complex non-convex geometric shape are used to protect harbour walls, breakwaters and shore earthworks. So, it is scientific significance and application prospects to study mechanical properties of granular materials under impact loading.

Especially, the buffering capacity is a fundamental issue for the dynamics of granular media[11]. However the energy dissipation and dynamic response during impact process of non-convex granular matter are still not well understood in both experiment and simulation. In this paper, based on the EDEM software platform, simulation of U-shaped granular bed under vertical impact loading is performed. The buffering capacity of U-shaped particles are analyzed by simulating different aspect ratios of particles.

\section{Numerical simulation}

To study the influences of non-convex particles shape on buffering capacity of granular system under impact load, three-dimensional discrete element method (DEM) with the soft contact approach is carried out to model the impacting process of spherical projectile on non-convex particle beds. Simulations in this study are performed by commercial software EDEM, the engineering simulation platform developed by DEM-Solutions Company. The motion of particles follows Newton's second law. The simplified Hertz-Mindlin model (Hertz-Mindlin no-slip model) which could make a good representation of the particle behavior during collisions, is employed to calculate the contact force between particles. Normal force and tangential force can be described in the form of nonlinear spring combined with dashpot, however the viscous forces such as liquid force were not considered here.

The multi-element method is used to represent the U-shaped particles as non-convex particles similar with previous article[6]. It is composed of several identically sized spherical elements overlapping each other as

\footnotetext{
*Corresponding author: zq_2012@,126.com
} 
shown in Fig.1. The diameter of a single unit is $2.8 \mathrm{~mm}$ and the overlap is $1.4 \mathrm{~mm}$. There are 9 different proportions of length to width for U-shaped particle, which is ranged from $0.25: 1$ to $1.417: 1$ in this work. In the multi-element method, contact detection between particles is sphere-based, which leads to the figuring of contact forces between U-shaped particles can be conducted with a standard discrete element algorithm for spherical particle.

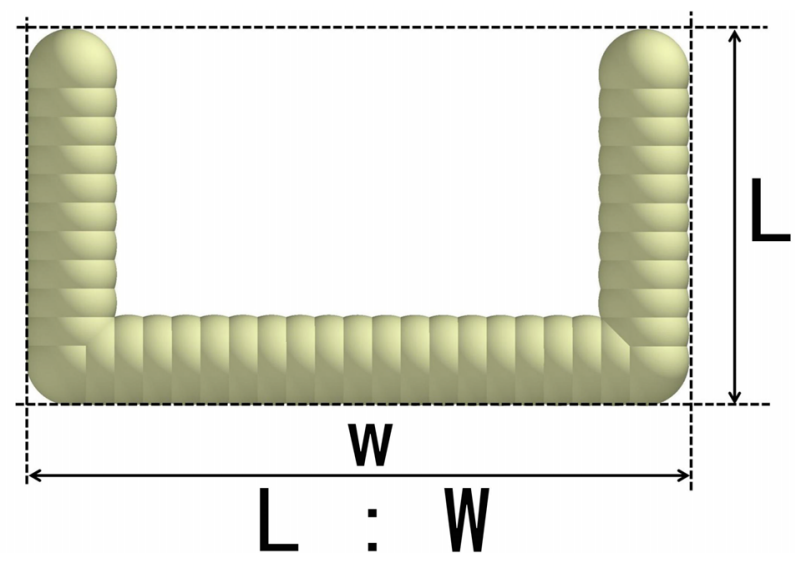

(a) U-shaped particle
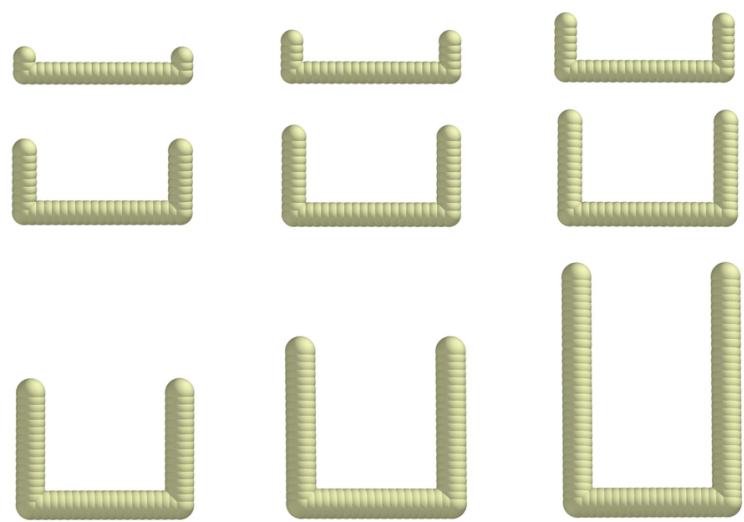

(b) different aspect ratio $\mathrm{L} / \mathrm{W}$

Fig. 1. U-shaped particles with different aspect ratio L/W generated by multi-element method

The cylindrical container is $80 \mathrm{~mm}$ in diameter and $200 \mathrm{~mm}$ in height. The main computational parameters in the DEM simulation are listed in Table 1. Firstly, U-shaped particles are generated randomly at the top of the cylindrical container. Secondly, all the beads fall into the container by rain-fall method. Then a rigid circular board appears above the surface of the granular bed closely and moves downwards $1 \mathrm{~mm}$ to flat the surface. When the velocity of particles is observably small, we remove the board. At last a spherical projectile with diameter of $14 \mathrm{~mm}$ generates above the surface of granular bed and impacts the bed with constant velocity $1 \mathrm{~m} / \mathrm{s}$ as shown in fig.2. For different aspect ratio of U-shaped particles, the total mass of the granular bed is constant.
Table 1. Main computational parameters in DEM simulation

\begin{tabular}{|c|c|c|}
\hline Parameters & Unit & Value \\
\hline Projectile poisson ratio & & 0.31 \\
\hline Projectile shear modulus & $\mathrm{GPa}$ & 4 \\
\hline Projectile density & $\mathrm{g} / \mathrm{cm}^{3}$ & 7.85 \\
\hline Projectile diameter & $\mathrm{cm}$ & 14 \\
\hline U-shaped particle poisson ratio & & 0.215 \\
\hline U-shaped particle shear modulus & $\mathrm{GPa}$ & 0.1 \\
\hline U-shaped particle density & $\mathrm{g} / \mathrm{cm}^{3}$ & 2.65 \\
\hline Coefficient of friction & & 0.5 \\
\hline Coefficient of restitution & & 0.5 \\
\hline Time step & $\mathrm{s}$ & $1 \mathrm{e}-6$ \\
\hline
\end{tabular}

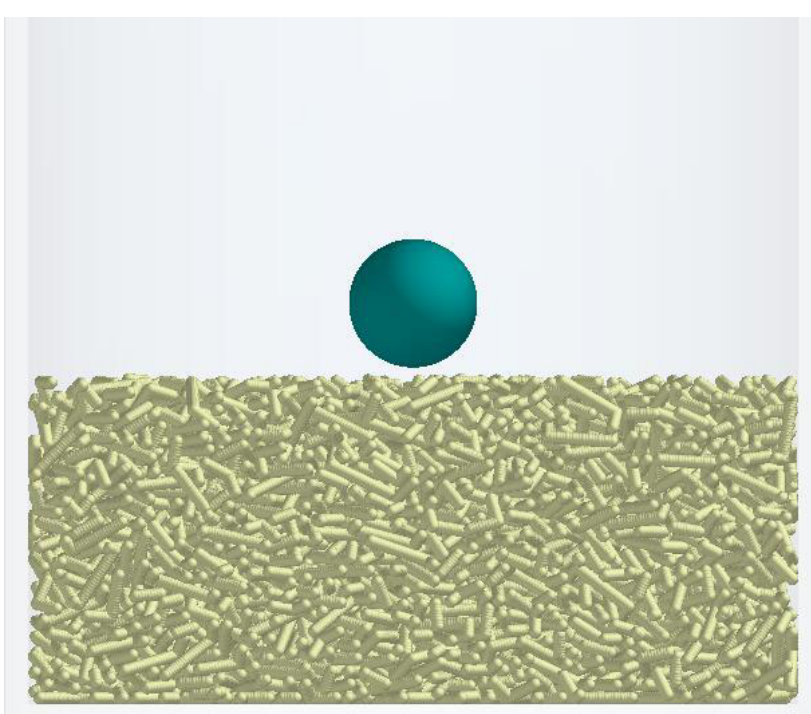

Fig. 2. The sketch map of simulation.

\section{Numerical results}

The first peak of impact force directly reflects the impact absorbing effect of granular materials. Therefore the compressive force on the base plate of the container is utilized to quantify the buffering capacity of non-convex particles as shown in Figure.3. The impact force of the base plate is obtained by subtracting the gravity of the granular bed from the total pressure on the base plate. The curve usually contains two parts, a large peak at beginning and a tail of small fluctuation wave continuing several milliseconds. The tail is not shown here. The results display that the impact load peaks decrease obviously with increase of particle aspect ratio from 0.125 to 0.792 . Meanwhile the duration time grows with the increase of aspect ratio. There is no significant difference between granular beds in which aspect ratio is larger than 0.8 . The negative value in the curve indicates that the particle system will oscillate in the process of impact. Then the oscillation results in a certain vibration 
of the base plate of the container so that sometimes the compressive force could go negative in Fig. 3. The simulation results are consistent with the reported experimental result[11].

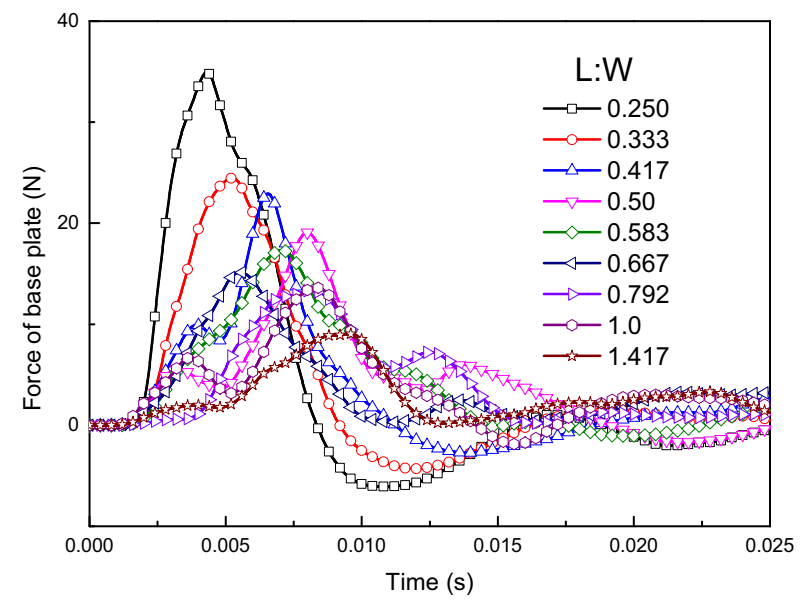

Fig. 3. Compressive force of base plate versus time under different granular aspect ratio.

The relationship between the compressive force peak and granular aspect ratio is plotted in Figure 4. Each line is obtained by average of five times simulation results. The dot line expresses the compressive force on base plate for the sample of spherical particles with diameter of $2.8 \mathrm{~mm}$ which total mass of granular bed is equal to the mass of U-shaped particles. It implies that the buffer capacity of high aspect ratio U-shaped particles is better than spherical particle with equal mass.

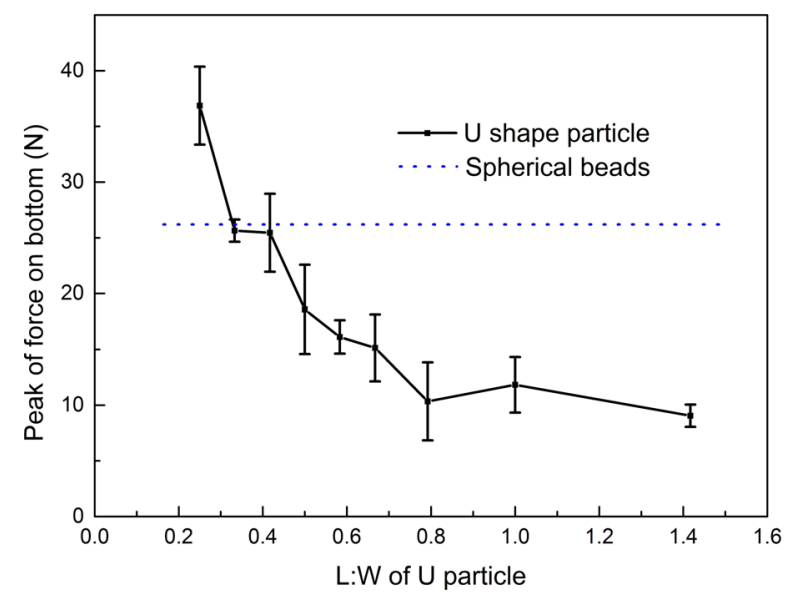

Fig. 4. The relationship between the compressive force peak and granular aspect ratio (the dot line represents compressive force peak of spherical beads)

\section{Discussion}

It is well known that sliding friction between particles is the main mechanism to dissipate energy of granular system. Prior study[12] has suggested non-spherical particles possess a higher effective friction, in comparison to spherical particles with the same inter-particle friction coefficient, due to suppression of rolling. On account of the entanglement between particles and the self-locking effect, the mechanism of the friction between non-convex particles is still not clear. We try to explain the influence of the non-convex particle shape on the cushioning properties of the particle system from the view of inter-friction.

Figure. 5 shows the variation of the average contact number during the impact process for different aspect ratio of U-shaped particles. The contact judgment algorithm of non-convex particles is the same as that of spherical particles. When two particles overlap, a contact is created. Once a contact has been detected, contact properties (such as particle prototype, size, and relative velocity) are passed to the contact model to calculate the force. It shows that for the low aspect ratio samples, the average contact number of granular bed reduces by nearly thirty percent in the duration of impact process; while for the high aspect ratio samples, the average contact number only reduces a small amount. Such behavior suggests that the entanglement between the non-convex particles and the inter-locking effect enable the system maintain relatively stable contact even suffering strong external disturbance.

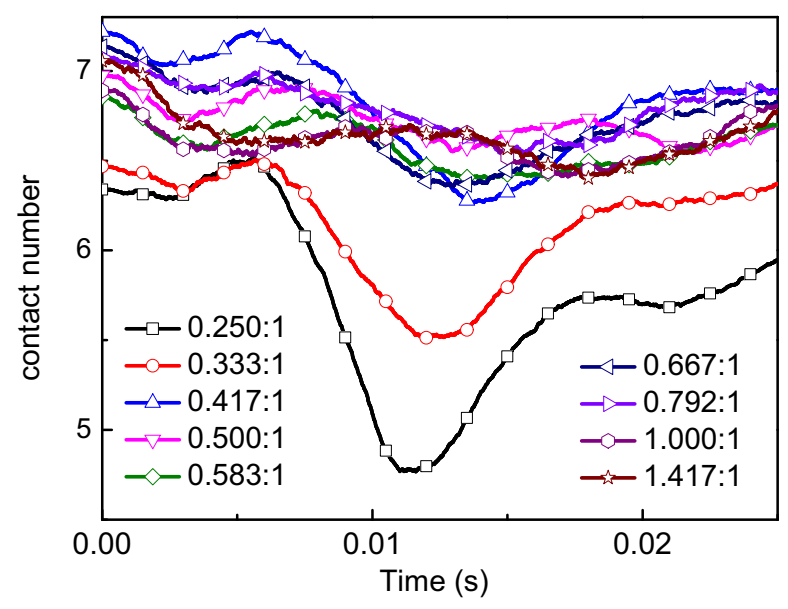

Fig. 5. Variation of the average coordination number during the impact process.

The entanglement of the non-convex particles and the inter-locking effect induce that the distribution of the internal non-uniform force chain is different from the spherical particles. The probability density function of the normal contact force between particles at the moment of collision is plotted in Fig.6. With the increasing of the aspect ratio of the sample, the probability of weak force is gradually decreasing, while the probability of the strong force appears gradually increasing. This feature of probability density function of normal force in the process of impact is similar. Such results imply that more stable contact and relative larger normal force lead to better energy dissipation ability for high aspect ratio non-convex particles. 


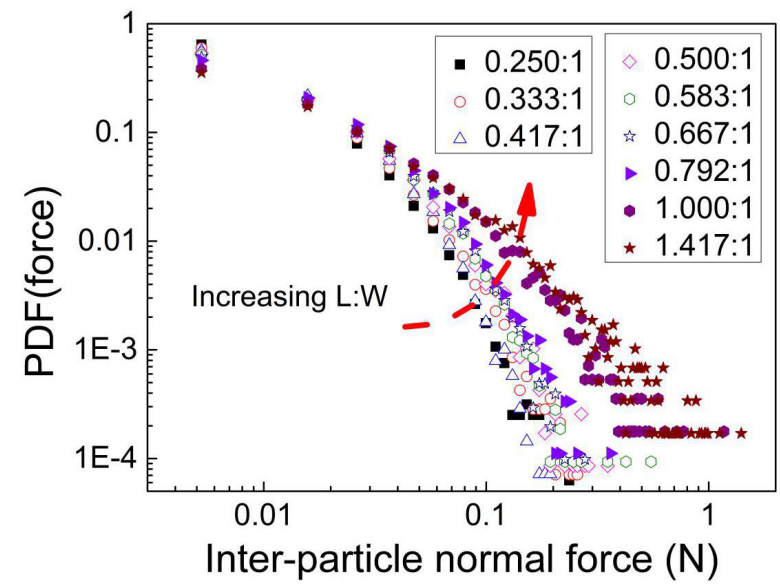

Fig. 6. Distribution of normal contact force for various aspect ratio particles

\section{Conclusion}

Granular material exhibits signally buffering capacity due to its dissipative nature. Numerical simulations show that the shape of non-convex particles notably influences the buffering capacity of granular bed. The buffering ability of high aspect ratio non-convex particles could be better than the spherical particles with equal mass. The shape of the non-convex particles significantly affects the contact number of granular system and probability density functions of inter-particle forces. We except this result could be helpful to study the ability of aleatory architectures[13] to withstand external disturbance.

We gratefully acknowledge the constructive suggestion of Prof. Shunying Ji (Dalian University of Technology) and Prof. Qicheng sun (Tsinghua University).

\section{References}

1. J.C. Ruiz-Suarez, Rep.Prog.Phys 76, 066601 (2013).

2. K. Van der Weele, Contemporary Physics 49, 157-178 (2008).

3. M.Z. Miskin, H.M. Jaeger, Soft Matter 10, 3708-3715 (2014).

4. S. van den Wildenberg, Y. Yang, X. Jia, Granular Matter 17, 419-426 (2015)

5. E. Brown, A. Nasto, A.G. Athanassiadis, H.M. Jaeger, Phys.Rev.Lett 108, 108302 (2012).

6. N. Gravish, S.V. Franklin, D.L. Hu, D.I. Goldman, Phys.Rev.Lett 108, 208001 (2012).

7. A. Seguin, Y. Bertho, P. Gondret.J.Crassous.EPL 88, 44002 (2009).

8. M. Nishida, Y. Tanaka, Granular Matter 12, 357-368 (2010).

9. J. Huang, S. Xu, S. Hu, INT.J.IMPACT.ENG 59, 1-10 (2013).
10. M. Omidvar, M. Iskander, S. Bless, INT.J.IMPACT. ENG 93, 211-221 (2016).

11. S. Ji, X. Chen, P. Li,Y. Yan, Powders and Grains 2013, 401-404 (2013).

12. H. Chen, Y.L. Liu, X.Q. Zhao, Y.G. Xiao,Y. Liu, Powder Technology 283, 607-617 (2015).

13. S. Keller1, H.M. Jaeger, Granular Matter 18, 29(2016) 